\title{
Control de brazo electrónico usando señales electromiográficas
}

\author{
Electronic control arm using electromyographic signals
}

Controle de braço eletrônico usando sinais eletromiográficos

Fecha de Recepción: 06 de Septiembre de 2014

Fecha de Aceptación: 23 de Diciembre de 2014

\author{
Jorge Andrés García-Pinzón* \\ Luis Enrique Mendoza** \\ Elkin Gregorio Flórez ${ }^{\star \star *}$
}

\section{Resumen}

Los trabajos enfocados en la extracción de patrones en señales electromiográficas (SEMG) han venido creciendo debido a sus múltiples aplicaciones. En este artículo se presenta una aplicación en la cual se implementa un sistema electrónico para el registro de las SEMG de la extremidad superior en un sujeto, con el fin de controlar de forma remota un brazo electrónico. Se realizó una etapa de preprocesamiento de las señales registradas, para eliminar información poco relevante, y reconocimiento de zonas de interés; enseguida se extraen los patrones y se clasifican. Las técnicas utilizadas fueron: análisis wavelet (AW), análisis de componentes principales (ACP), transformada de fourier (TF), transformada del coseno discreta (TDC), energía, máquinas de soporte vectorial (MSV o SVM) y redes neuronales (RNA). En este artículo se demuestra que la metodología planteada permite realizar un proceso de clasificación con un rendimiento superior al 95\%. Se registraron más de 4000 señales.

Palabras clave: Brazo electrónico, Electromiografia, Extracción de patrones, MSV, RNA, Transformada wavelet.

\section{Abstract}

The studies focused in pattern extractions of electromyography signals (SEMG) has been growing, due to their multiple applications. This paper presents an electronic system implementation for the SEMG recording of a subject upper extremity in order to remotely control an electronic arm. Initially, we performed a signals preprocessing, to remove the less important information and to recognize the interest areas. Then the patterns were

* M.Sc. Universidad de Pamplona (Pamplona - Norte de Santander, Colombia). jorgepinzon1101@gmail.com.

** M.Sc. Universidad de Pamplona (Pamplona - Norte de Santander, Colombia). luis.mendoza@unipamplona.edu.co.

*** Ph.D. Universidad de Pamplona (Pamplona - Norte de Santander, Colombia). eflorez@unipamplona.edu.co. 
extracted and classified. The techniques used were: The wavelet analysis (AW), the principal components analysis (PCA), the Fourier transformed (FT), the discrete cosine transformed (DCT), the support vector machines (SVM) and the artificial neural networks (ANR). In this paper we demonstrated, that the methodology stated, allows to realize a process of classification with a superior performance to $95 \%$. There were recorded more than four thousands signals.

Keywords: Electronic Arm Control, Electromyography, ANR, SVM, Patterns Extraction, Wavelet Transformed.

\section{Resumo}

Os trabalhos enfocados na extração de padrões em sinais eletromiográficos (SEMG) têm crescido devido a suas múltiplas aplicações. Neste artigo se apresenta uma aplicação na qual se implementa um sistema eletrônico para o registro das SEMG da extremidade superior em um sujeito, com o fim de controlar de forma remota um braço eletrônico. Realizou-se uma etapa de pré-processamento dos sinais registrados, para eliminar informação pouco relevante, e reconhecimento de zonas de interesse; em seguida se extraem os padrões e se classificam. As técnicas utilizadas foram: análise wavelet (AW), análise de componentes principais (ACP), transformada de fourier (TF), transformada do coseno discreta (TDC), energia, máquinas de suporte vectorial (MSV ou SVM) e redes neuronais (RNA). Neste artigo se demonstra que a metodologia planteada permite realizar um processo de classificação com um rendimento superior a 95\%. Registraram-se mais de 4000 sinais.

Palavras chave: Braço eletrônico, Eletromiografia, Extração de padrões, MSV, RNA, Transformada wavelet.

Cómo citar este artículo:

[1] J.A. García-Pinzón, L.E. Mendoza \& E. G. Flórez, “Control de brazo electrónico usando señales electromiográficas”, Fac. Ing., vol. 24 (39), pp. 71-83, Mayo-Ago. 2015. 


\section{INTRODUCCIÓN}

Las señales electromiográficas (SEMG) son datos o señales eléctricas que muestran el comportamiento del sistema biológico muscular que compone el cuerpo humano; hoy en día, dichas señales, son utilizadas en sistema biomédicos y de bio-ingeniería, ya que sus ventajas y aplicaciones representan un aporte importante [1-3]. Entre las aplicaciones con mayor impacto usando señales electromiográficas se tienen: seguimiento del rendimiento de deportistas, control de prótesis, control de equipos mecánicos y electrónicos de alta complejidad y sistemas de teleoperación [4-7]. En la actualidad, el control de sistemas electrónicos usando SEMG ha abierto un campo de investigación bastante amplio; es así como diferentes trabajos se han desarrollado en esta área [8-12] y enfocan sus investigaciones en el control de prótesis, control de la marcha y uso de técnicas avanzadas para el reconocimiento de patrones en SEMG [13-14]. Este trabajo plantea una metodología con el fin de utilizar las SEMG para controlar un sistema electrónico y, en futuras investigaciones, manipular sustancias químicas por medio de sistemas mecánico-electrónicos. Es importante mencionar que se debe tener en cuenta para este tipo de trabajos la parte fisiológica y el posicionamiento de los electrodos en la extremidad superior [15]; estas dos premisas son importantes, ya que si no se mantienen constantes durante la toma de datos se podrían generar problemas en el control del sistema electrónico.

Así mismo, las técnicas de procesamiento de señales utilizadas para el procesamiento de datos EMG han venido creciendo, ya que su utilidad es vista en aplicaciones como extracción de patrones de marcha, cuantificación de angulación de las articulaciones y clasificación de problemas o patologías, entre otras [12-13]. Por otro lado, las técnicas de procesamiento de datos encierran también aplicaciones tales como: eliminación de ruido, detección de zonas de interes, segmentación de información, minería de datos y clasificación. En este artículo se presenta el uso y comparación de técnicas como: TF, TDC, Energía y TW, con fines de eliminación de ruido y extracción de patrones. Estas técnicas permiten el desarrollo de una clasificación usando RNA y SVM [16-19]. Las señales a clasificar representan movimientos como: bíceps, hombro, mano y la posición neutral, definida como quieto.

El uso de las técnicas de procesamiento en el procedimiento planteado en este artículo se justifica, ya que la amplitud de la SEMG presenta una morfología aleatoria y poco reproducible; por lo general se evidencia como una función gaussiana [17]; esto indica que para el mismo paciente se pueden obtener patrones diferentes del mismo movimiento. Dicha amplitud de la señal viene dada en microvoltios, lo que indica que se debe tener un cuidado especial a la hora de registrar la señal y no perder información relevante.

Finalmente, en este trabajo se demuestra que se obtienen mejores resultados usando máquinas de soporte vectorial que redes neuronales, ya que los porcentajes de clasificación para el control de brazo son más exitosos. También se demostró que es mejor usar SVM que RNA, por su rendimiento en tiempo. Por otro lado, se consiguieron resultados eficientes usando el análisis wavelet, ya que con dicha técnica los patrones encontrados permitieron obtener porcentajes de acierto más elevados, en comparación con técnicas como Fourier y discreta del coseno.

\section{Metodología}

La metodología de la investigación se llevó a cabo en tres fases, como se muestra en la Fig. 3. 


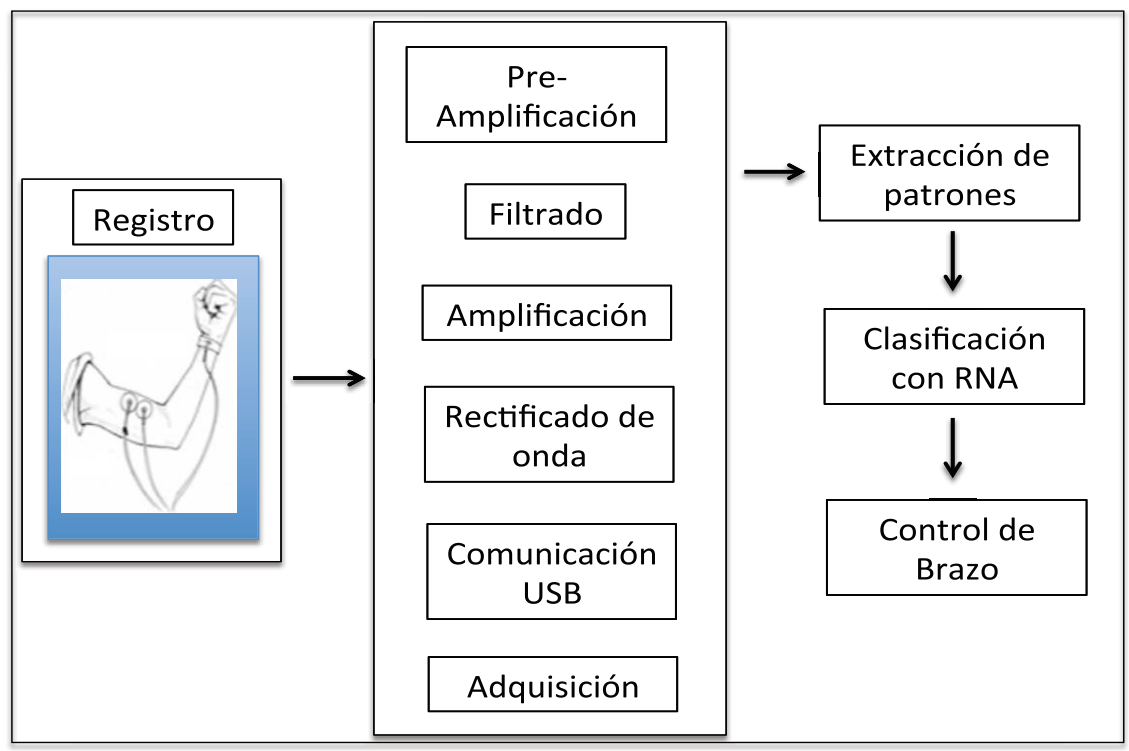

Fig. 3. Diagrama de bloques de la metodología

A continuación se describen las etapas de la metodología.

\section{A. Adquisición}

El circuito implementado para adquirir y acondicionar la SEMG consta de las siguientes etapas: preamplificación, filtrado, amplificación, rectificación de onda completa y comunicación USB. Se realizó un sistema de adquisición de dos canales: el primero, conectado al antebrazo (llamado de aquí en adelante canal A), y segundo, conectado al brazo (llamado de aquí en adelante canal B), con el fin de tener mejor resolución de respuesta. Se utilizaron 4 movimientos para controlar el sistema: mano, bíceps, hombro y quieto. Para la implementación de adquisición se usó el dispositivo INA115, que cumple satisfactoriamente la tarea para la captura. Para digitalizar los datos en la interfaz se optó por utilizar el protocolo USB, dos tipos de transferencias que emulan el puerto serial, una conocida como CDC y la otra conocida como propósito general (Bulk Transfer); esta última cumple con las expectativas esperadas y fue la seleccionada, ya que transfieren muestras a mayor velocidad que CDC. Finalmente, se usó una frecuencia de muestreo de $1.5 \mathrm{Khz}$.

\section{B. Extracción de patrones}

La fase de extracción de patrones de las SEMG incluye la eliminación de ruido, el reconocimiento de zonas de interés y la búsqueda de patrones diferenciadores en cada grupo de señales. Las tres fases anteriores lograron mejorar la calidad de la información de las SEMG para el proceso de clasificación. En el estado original de las señales se hizo difícil realizar un proceso de clasificación, ya que los patrones eran similares entre los grupos.

Las estructuras matemáticas resumidas de las técnicas utilizadas son descritas a continuación.

1) Análisis de componentes principales (ACP): $\mathrm{El}$ objetivo es transformar un conjunto dado de datos de dimensión a otro conjunto de datos de menor dimensión con la menor pérdida de información posible, utilizando para ello la matriz de covarianza de (1), (2).

$$
\begin{aligned}
& \operatorname{cov}(X)=\frac{X^{T} X}{n-1} \\
& \operatorname{cov}(X) P_{a}=\lambda_{a} P_{a}
\end{aligned}
$$

Donde $\lambda_{a}$ es el valor propio asociado al vector propio $P_{a}$ 
2) La transformada de Fourier (TF): Obtiene las componentes de frecuencia de una señal cualquiera, tal como se muestra en (3).

$X(f)=\int_{-\infty}^{\infty} x(t) e^{-2 \pi f t} d t$

Donde: $x(t)=$ Señal de prueba

$e^{-2 \pi f t}=$ Exponencial complejo

$X(f)=$ Espectro

3) Transformada Discreta del coseno (TDC): Es un tipo de transformada real y ortogonal, tal como se muestra en (4).

$F(k)=C(k) \sum_{n=0}^{N-1} f[n] * \cos \frac{\pi(0.5+n) k}{2 N}$

Donde: $f[n]=$ Representa la secuencia de muestras discretas

$F(k)=$ Coeficientes de la TDC

$\mathrm{N}=$ Número de coeficientes del TDC

4) La transformada de wavelet (TW): Se define como un análisis multiresolucional de una función $f(t)$, el cual descompone la señal original en un conjunto de funciones $W_{f}(s, \tau)$ que forman los coeficientes wavelets, como se observa en (5).

$W_{f}(s, \tau)=\int f(t) \Psi_{s, \tau}(t) d t$

Las Wavelets son generadas a partir de la traslación y cambio de escala de una misma función wavelet $\Psi_{s, \tau}(t)$, llamada la "Wavelet madre", y se define en (6).

$\Psi_{s, \tau}(t)=\frac{1}{\sqrt{s}} \Psi\left(\frac{t-\tau}{s}\right)$
Donde $s$ es el factor de escala y $\tau$ es el factor de traslación. En este trabajo se usó la wavelet madre db7 y niveles de descomposicion 3, 4, 5 y 6 .

5) Análisis de energía (E): Cantidad de información presente en una señal. Para el análisis de energía se utilizó (7).

$E=\max _{i}\left(\frac{\sum X^{2}}{n}\right)$

Donde: $1 \leq i \leq 20$

\section{Clasificación}

Se realizó un algoritmo usando SVM y RNA para clasificar los cuatro tipos de señales adquiridas y así controlar de forma precisa el movimiento del brazo electrónico. En este caso se usó una red probabilística multiclases, con 20 entradas, y un conjunto de entrenamiento de 200 señales, es decir, 50 señales de cada clase. Por otro lado, en cuanto a SVM, se usó un kernel función de base radial (RBF), con un parámetro de holgura de 0.45. Para obtener los parámetros de la SVM fue necesario realizar un número de 50 iteraciones con el propósito de conseguir el porcentaje de clasificación lo suficientemente robusto, usando como data de validación la misma data de entrenamiento.

\section{Control final}

La respuesta del sistema de clasificación se utiliza para manipular el control electrónico del circuito de potencia implementado; con este circuito (control final) se controlan las electroválvulas para posicionar el brazo. En las Fig. 4, Fig. 5, Fig. 6 y Fig. 7 se pueden observar las SEMG adquiridas de los 4 movimientos característicos y las posiciones que corresponden en el brazo electrónico. 


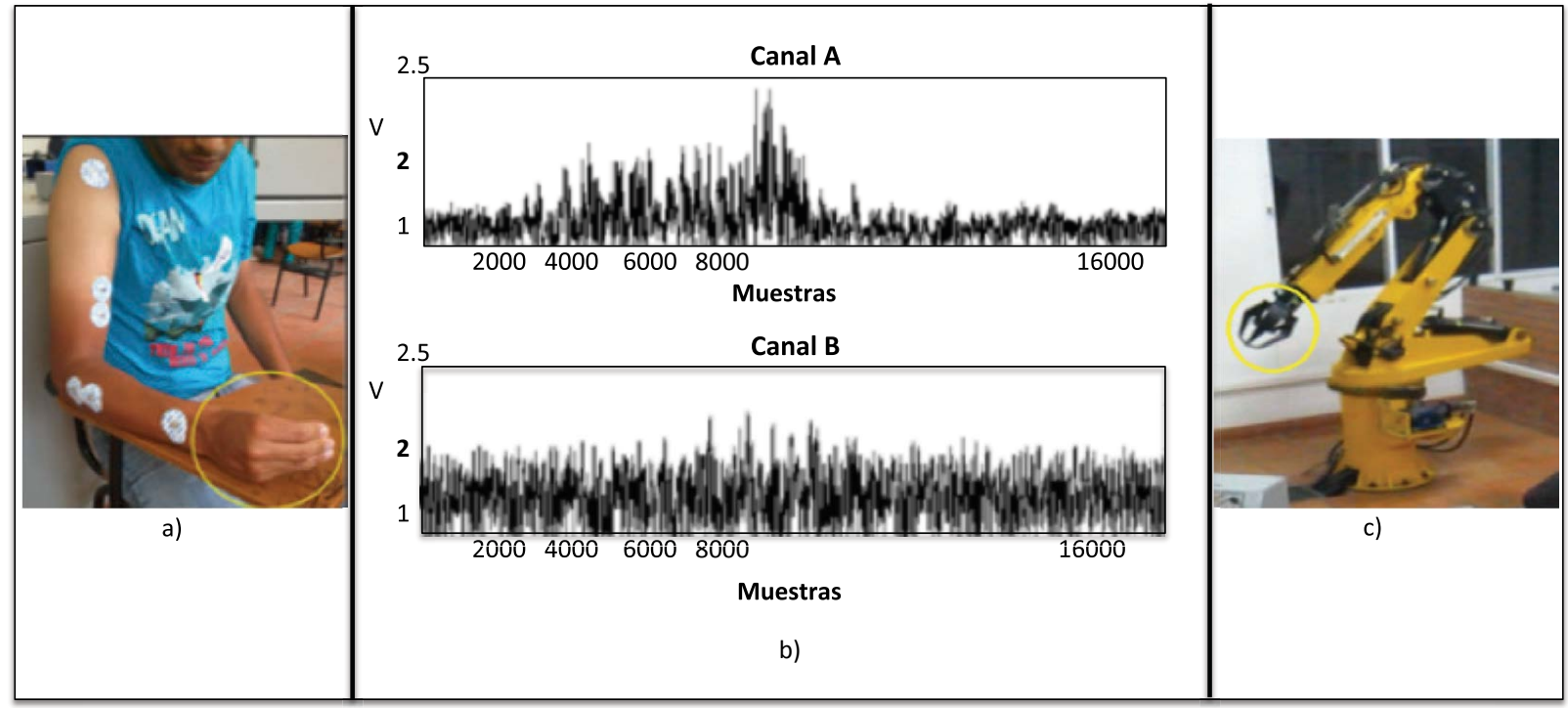

Fig. 4. a) Primer movimiento característico: contracción de la mano; b) Señal EMG registrada movimiento de la mano; c) Control de la pinza del brazo.

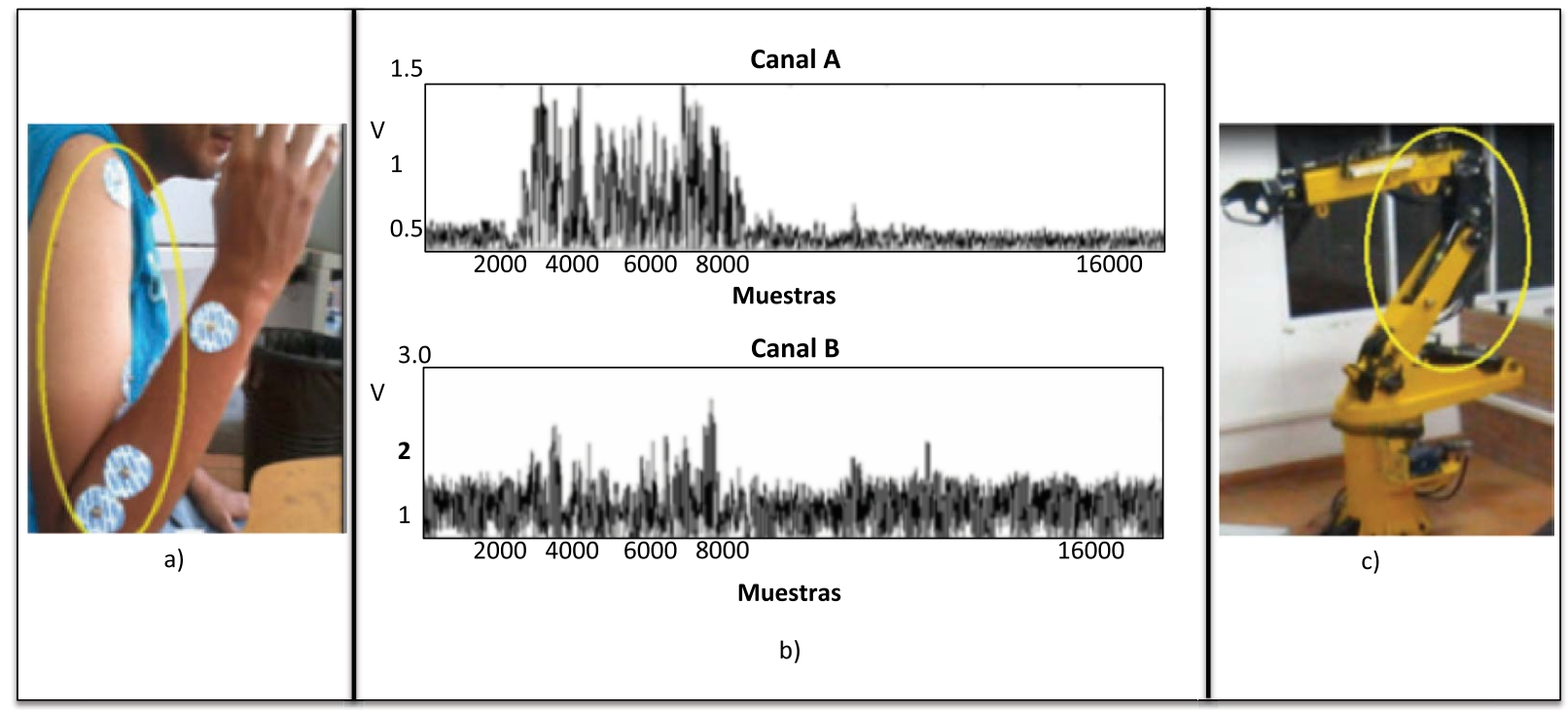

Fig. 5. a) Segundo movimiento característico contracción del bíceps; b) Señal EMG registrada movimiento de la bíceps; c) Control del cilindro del brazo. 


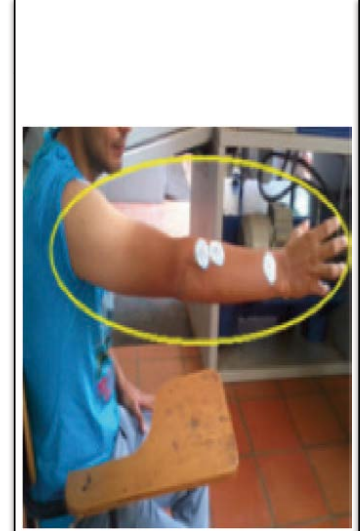

a)

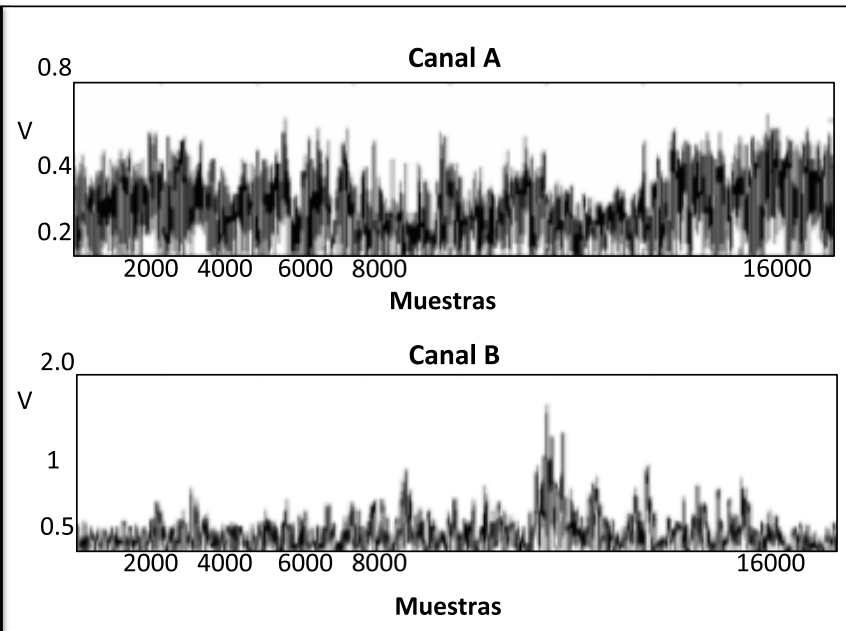

b)

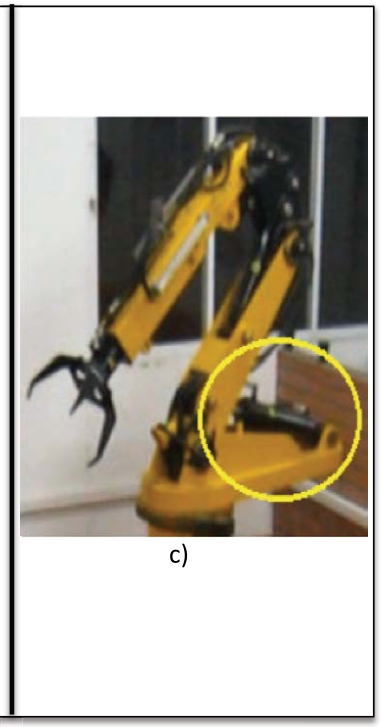

Fig. 6. a) Tercer movimiento característico flexión del hombro; b) Señal EMG registrada movimiento del hombro; c) Control del cilindro del brazo.

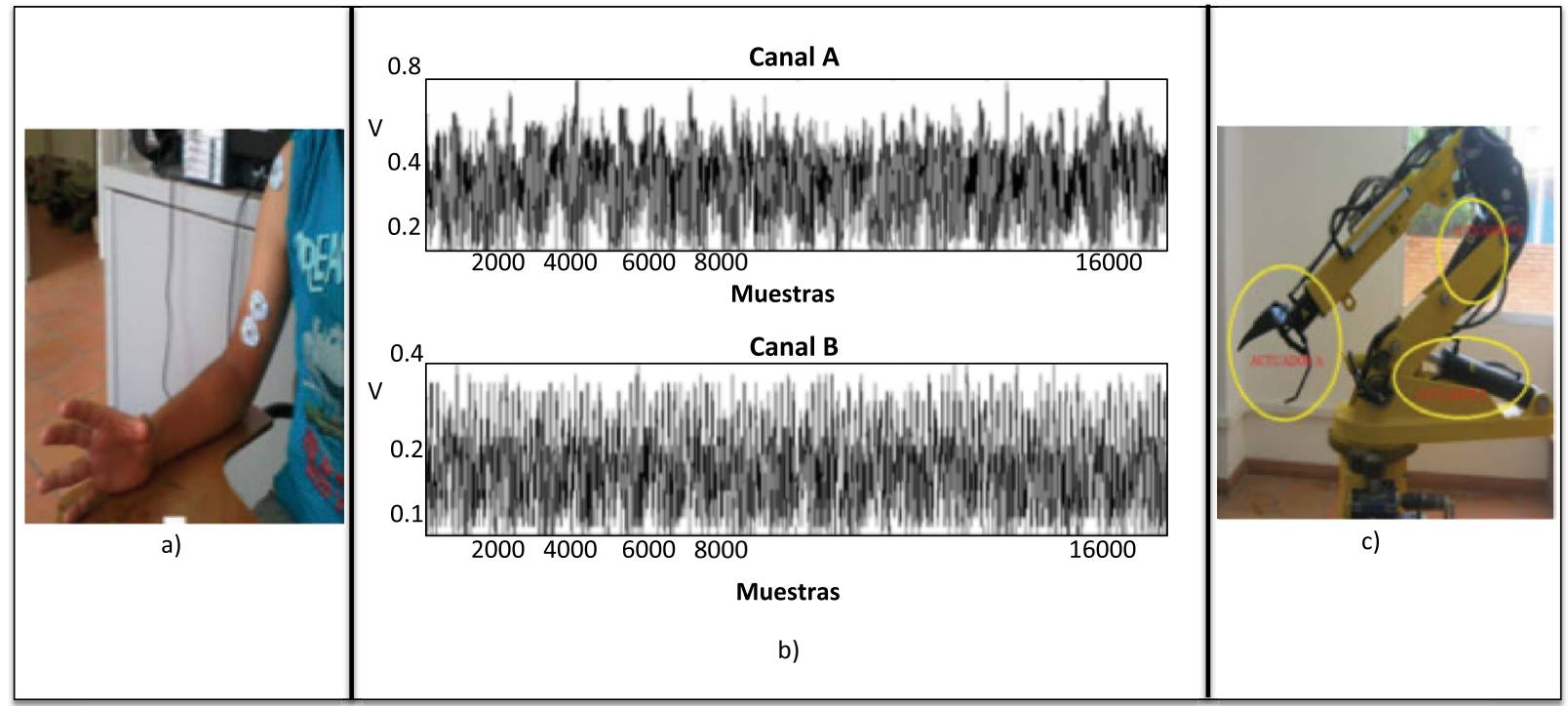

Fıg. 7. a) Posición inicial del miembro superior; b) Señal EMG registrada sin movimiento; c) Posición inicial del brazo hidráulico. 


\section{TABLA I}

\section{ClasificaCión DE LOS MOVIMIENTOS}

\begin{tabular}{c|c|c}
\hline $\begin{array}{c}\text { RNA y MSV Canal } \\
\text { A (Electrodos del } \\
\text { antebrazo) }\end{array}$ & $\begin{array}{c}\text { RNA y MSV Canal } \\
\text { B (Electrodos del } \\
\text { bíceps) }\end{array}$ & CLASIFICACIÓN \\
SALIDA & SALIDA & Quieto \\
\hline 1 & 1 & Bíceps \\
1 & 2 & Hombro \\
4 & 3 & Mano \\
\hline
\end{tabular}

En la Tabla I se muestran las combinaciones que se deben cumplir para reconocer cada uno de los movimientos; por ejemplo, para obtener el movimiento de bíceps, la RNA artificial del canal A debe tener una respuesta de 3, y la RNA del canal B debe tener una respuesta de 2. De no ser la respuesta indicada, el sistema hace caso omiso y no ejecuta ninguna acción. Este proceso permitió tener un sistema más robusto basado en dos canales.

\section{RESUltados}

A continución se muestran los resultados de la extracción de características de las SEMG de la extremidad superior. Las Fig. 9, Fig. 10, Fig. 11, Fig. 12, Fig. 13, Fig. 14, Fig. 15 y Fig. 16 muestran los resultados al aplicar cada una de las técnicas a las SEMG registradas, una vez ubicada la zona de interés. La zona de interés se ubica con el fin de no procesar data que no tiene informacion importante. Para el proceso de clasificación, una vez aplicada cada una de las técnicas de extracción de patrones, se tuvieron en cuenta los 20 picos con mayor amplitud. En este caso, la señal de color azul oscuro corresponde al movimiento de la mano; la señal de color verde, al movimiento del bíceps; la señal de color rojo, al movimiento del hombro, y la señal de color azul claro, sin movimiento. En las figuras anteriormente mencionadas se puede observar que los mejores resultados se obtuvieron usando wavelet y análisis de energía, ya que con Fourier y Coseno los patrones son muy similares entre grupos.

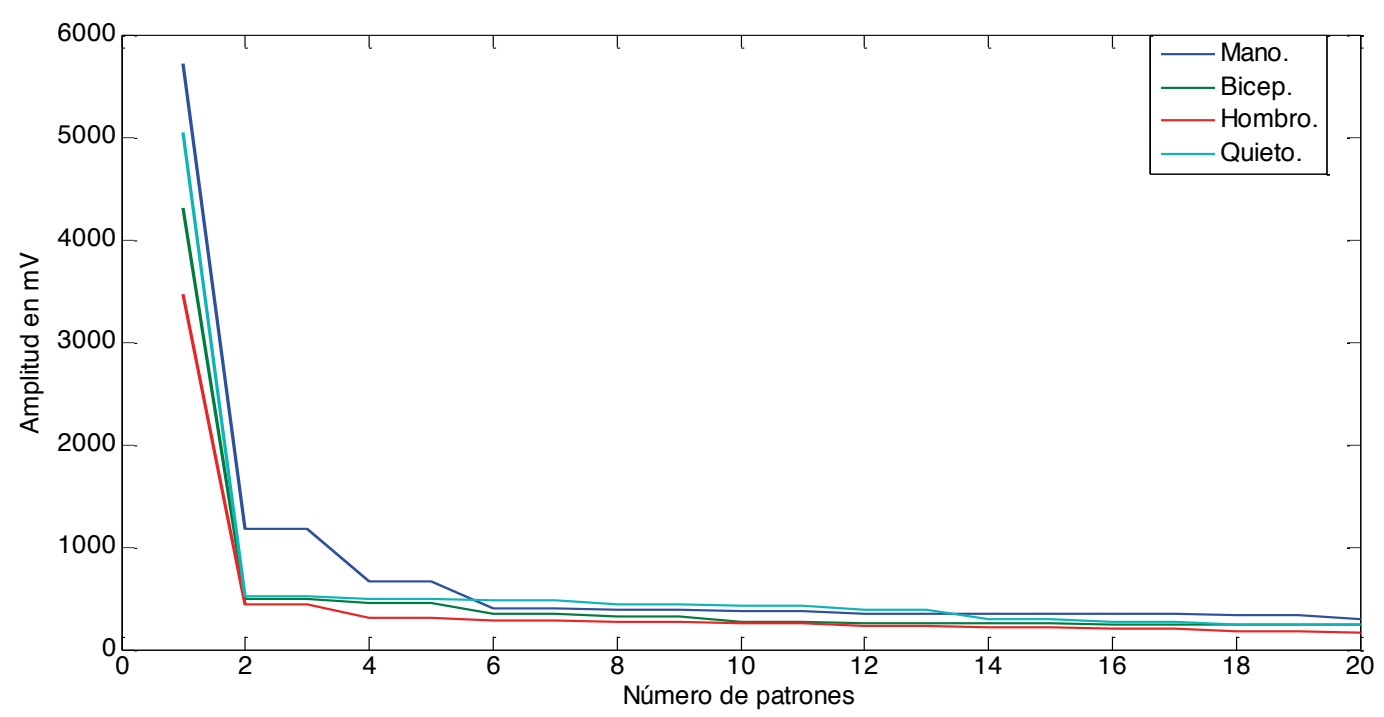

Fig. 9. La transformada de Fourier (FFT) del canal A, para los diferentes movimientos. 


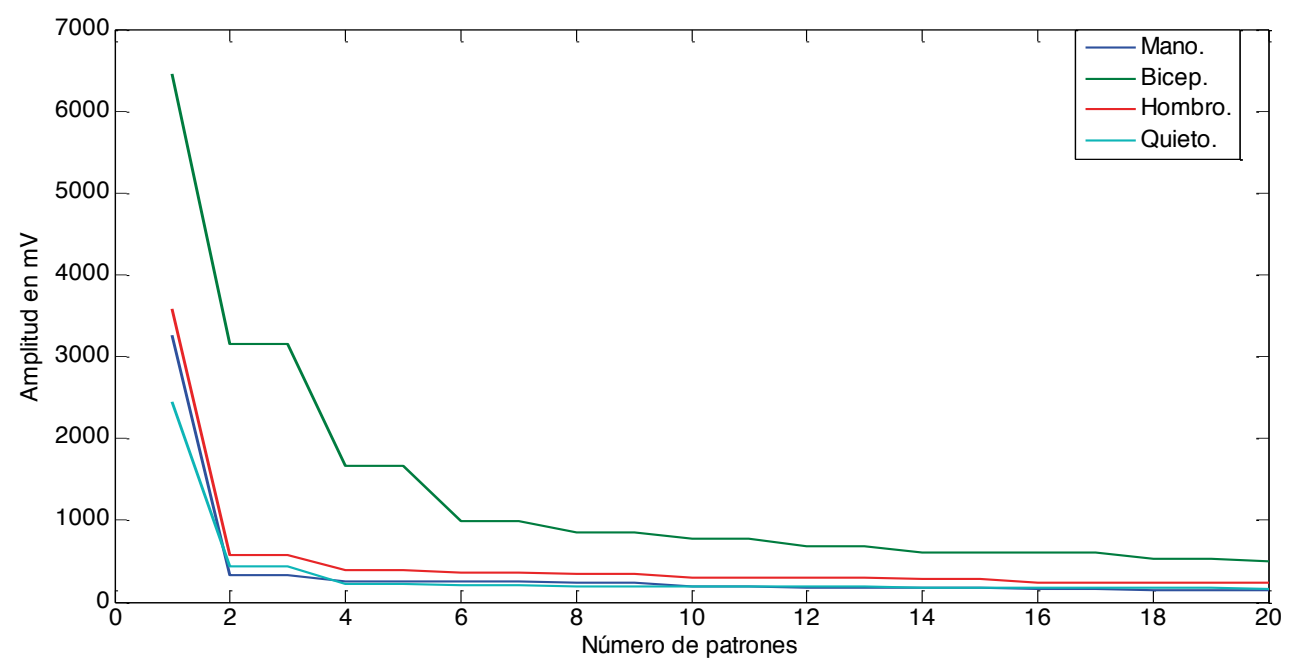

Fig. 10. La transformada de Fourier (FFT) del canal B, para los diferentes movimientos.

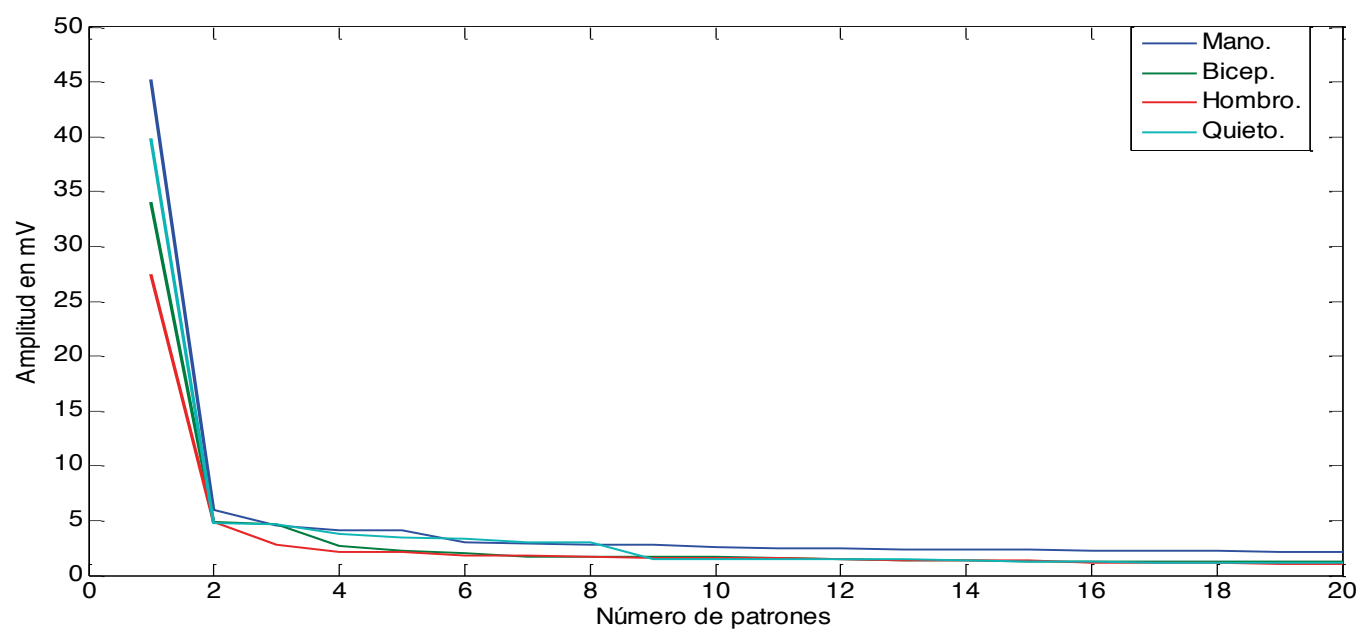

Fig. 11. La transformada discreta del coseno (DCT) del canal A, para los diferentes movimientos.

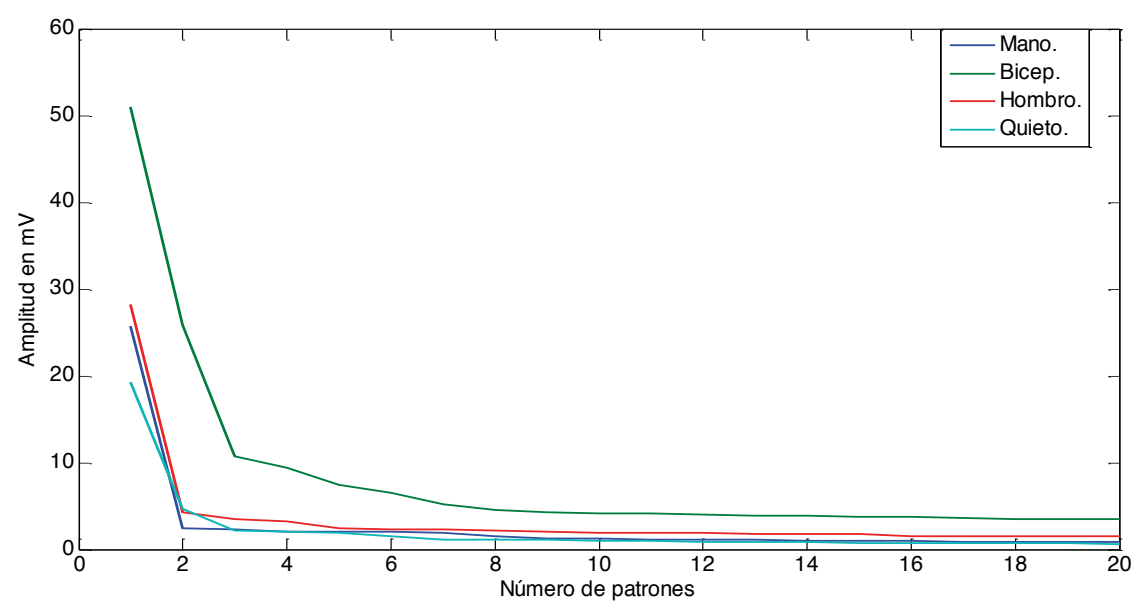

Fig. 12. La transformada discreta del coseno (DCT) del canal B, para los diferentes movimientos. 


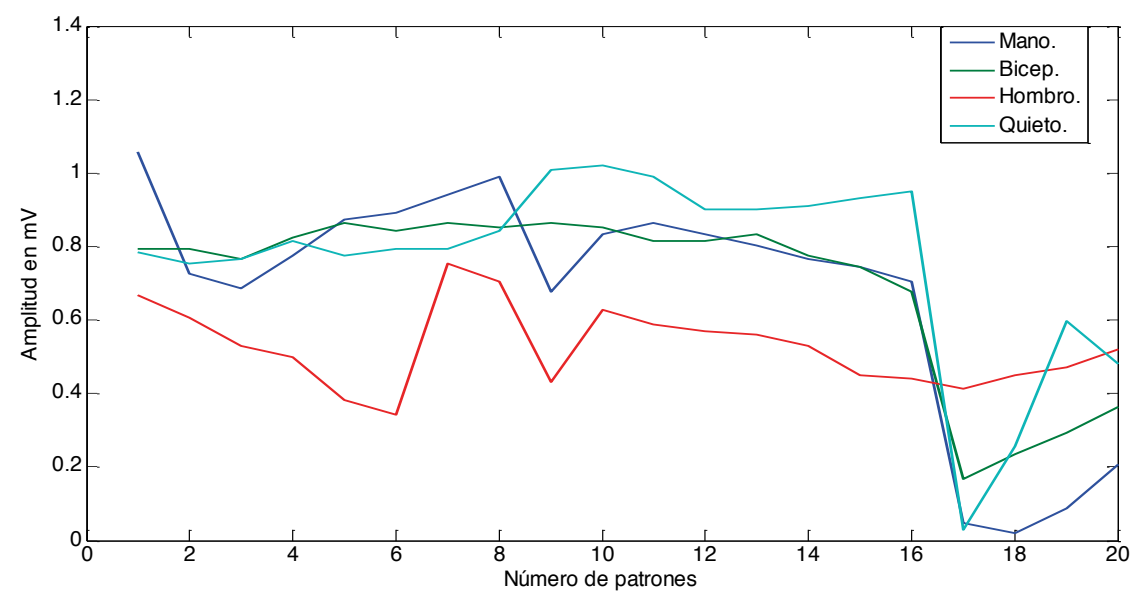

Fig. 13. La transformada de wavelet (TW) del canal A, para los diferentes movimientos.

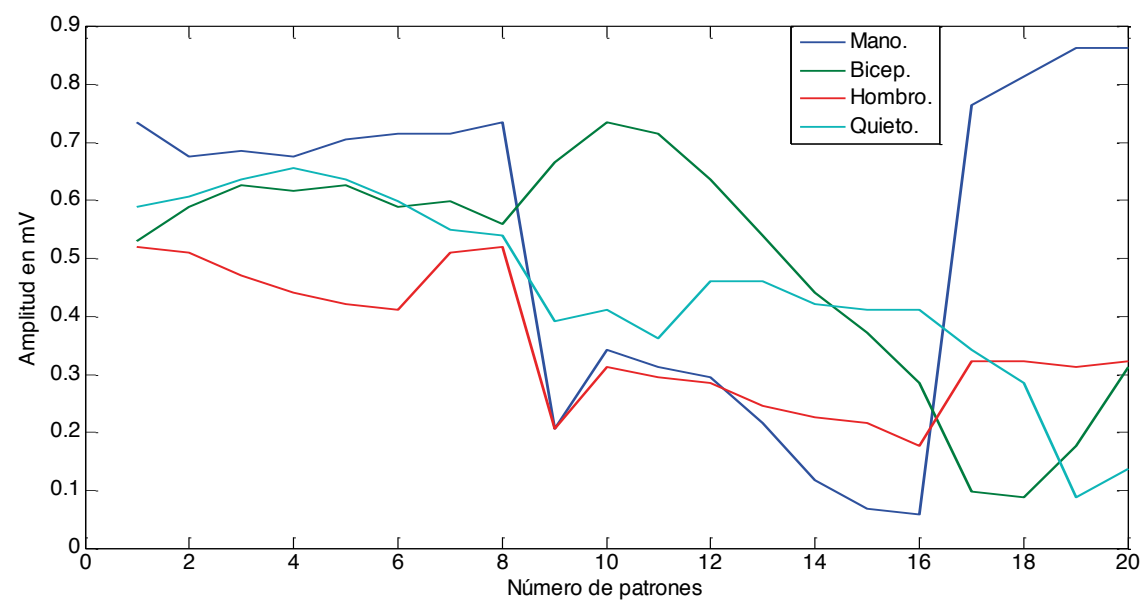

Fig. 14. La transformada de wavelet (TW) del canal B, para los diferentes movimientos.

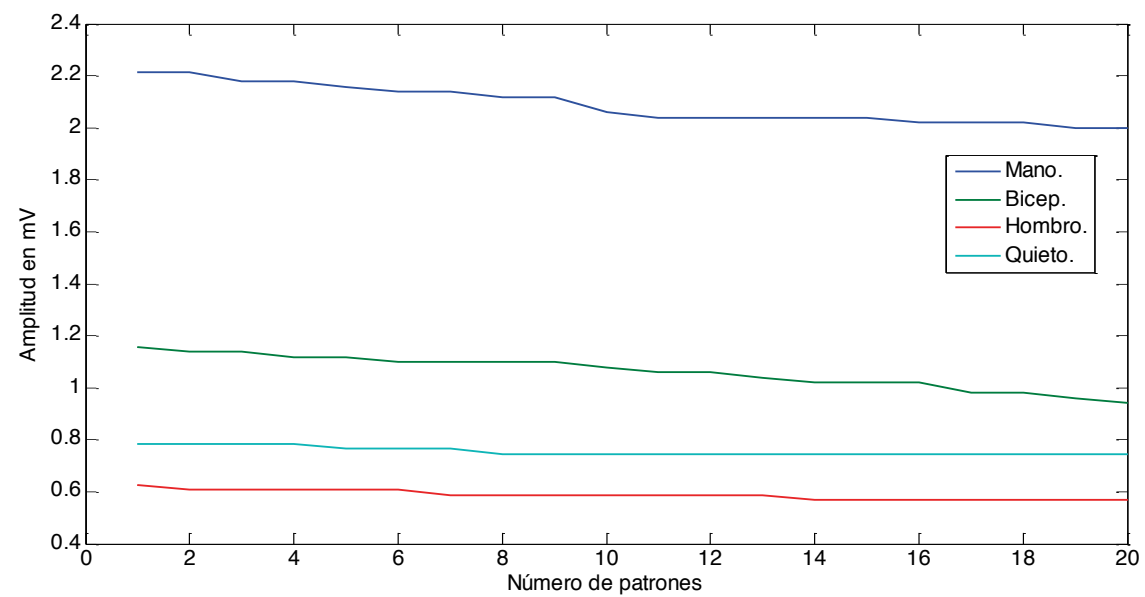

Fig. 15. Análisis de energía (E) del canal A, para los diferentes movimientos. 


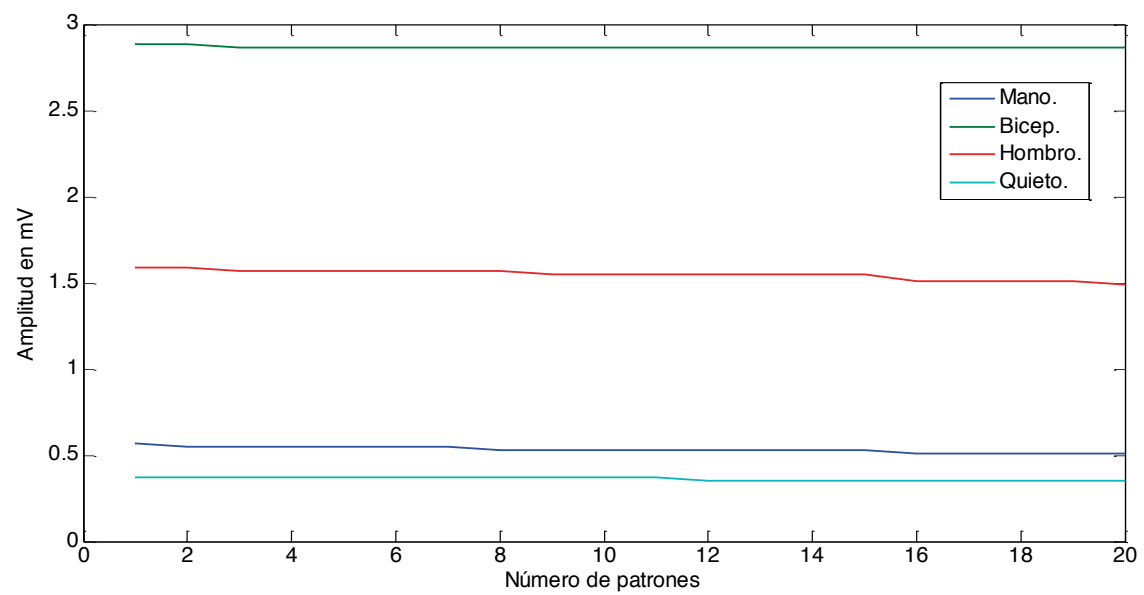

Fig. 16. Análisis de energía (E) del canal B, para los diferentes movimientos.

La Tabla II muestra una comparación que sustenta y resume por qué se seleccionó la energía y wavelet como parámetros de interés.

\section{TABla II}

\section{COMPARACIÓN DE LAS TÉCNICAS}

\begin{tabular}{|l|l|}
\hline $\begin{array}{l}\text { TDC } \\
\text { TF }\end{array}$ & $\begin{array}{l}\text { Su extracción de patrones para cada movimiento son similares. } \\
\text { Su extracción de patrones para cada movimiento son similares. }\end{array}$ \\
\hline WAVELET & $\begin{array}{l}\text { Su extracción de patrones para cada movimiento son similares, sin embargo, su nivel de } \\
\text { descomposición es diferentes. }\end{array}$ \\
\hline ACP & Tiempo de ejecución 10 segundos. \\
\hline ENERGÍA & La extracción de características es única. \\
\hline
\end{tabular}

Adicionalmente, se diseñó una interfaz gráfica (GUI) para visualizar las SEMG (ver Fig. 17). En dicha GUI se observa un ejemplo de las señales registradas, los

patrones reconocidos y el movimento que realiza el sistema.

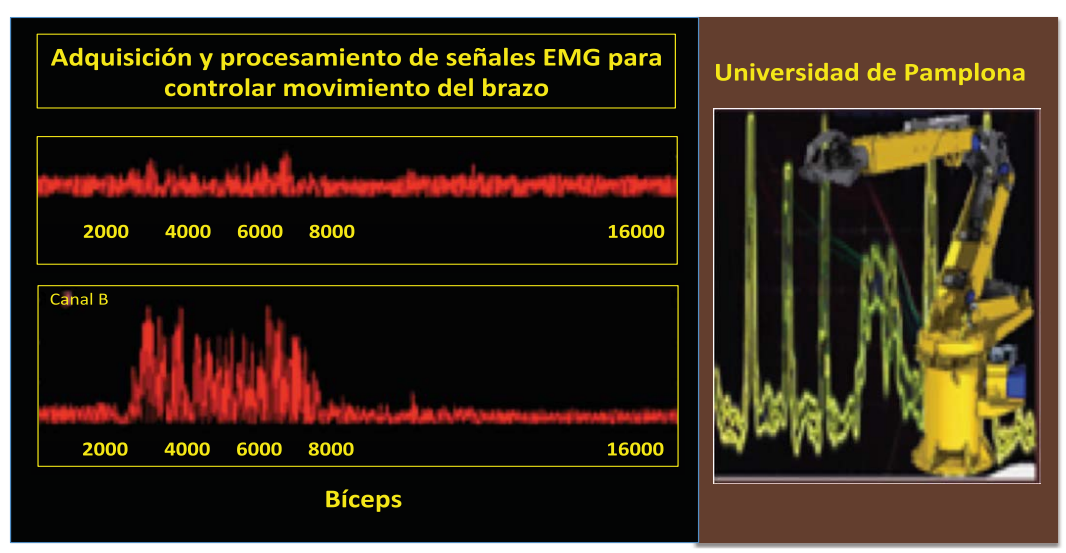

Fig. 17. Interfaz de adquisición y reconocimiento de las señales EMG. 
En cuanto a los resultados de clasificación, la Tabla III muestra los porcentajes de clasificación correcta obtenidos con RNA y SVM, teniendo como datos de entrenamiento los resultados de las técnicas TDC,
TF, Wavelet y energía. Es importante mencionar que para obtener este porcentaje se usó el error medio cuadrático.

\section{TABLA III}

\section{PORCENTAJES DE CLASIFICACIÓN CON LAS DIFERENTES TÉCNICAS}

\begin{tabular}{ccc}
\hline $\begin{array}{c}\text { Técnicas de extracción de } \\
\text { patrones }\end{array}$ & $\begin{array}{c}\text { \% Clasificación } \\
\text { RNA }\end{array}$ & $\begin{array}{c}\text { \% Clasificación } \\
\text { SVM }\end{array}$ \\
\hline TDC & 21.22 & 55.8 \\
\hline TF & 23.33 & 45.8 \\
Wavelet & 93.66 & 95.35 \\
Energía & 96.12 & 98.76 \\
\hline
\end{tabular}

El mayor porcentaje de clasificación correcto fue encontrado usando los parámetros obtenidos por wavelet y por el análisis de energía; estos datos fueron obtenidos usando 1000 señales de cada grupo. Los datos utilizados para validar el sistema fueron diferentes a los usados para entrenar las RNA y las SVM.
En cuanto a las pruebas de efectividad del sistema de clasificación, se realizaron usando 200 señales por cada movimiento, teniendo un total de 800 señales. Estas pruebas se realizaron 5 veces, para así poder tener un porcentaje de efectividad. En la Tabla IV se observan los resultados obtenidos en cuanto a la efectividad del clasificador, que obtuvo un $\%$ de efectividad del 96.4 , lo cual significa que las técnicas utilizadas son adecuadas para este tipo de señales.

\section{TABLA IV}

EFECTIVIDAD DEL SISTEMA

\begin{tabular}{|l|c|c|}
\hline CLASES & MUESTRAS & $\begin{array}{c}\text { DESVIACIÓN } \\
\text { ESTÁNDAR }\end{array}$ \\
\hline Mano & 991 & 0.0098 \\
\hline Bíceps & 990 & 0.055 \\
\hline Hombro & 987 & 0.0144 \\
\hline Quieto & 996 & 0.0085 \\
\hline Errores & 46 & \\
\hline Total señales & 4000 & \\
\hline Efectividad total & $\mathbf{9 6 . 4 \%}$ & \\
\hline
\end{tabular}

\section{Conclusiones}

Las SEMG tienen la característica de llevar en su morfología el comportamiento y los patrones de los movimientos del músculo del cuerpo humano, y además permiten conocer y encontrar posibles anormalidades en el sistema muscular. En esta investigación se registraron más de 6000 señales de los diferentes movimientos, las cuales se tomaron únicamente de la extremidad superior derecha. En cuanto al objetivo final de la investigación, se logró comprobar que usando un proceso de extracción de patrones con wavelet y análisis de energía se controló el sistema electrónico con un acierto máximo $98.76 \%$, usando como clasificador SVM. Estos resultados indican que es posible usar la metodología planteada en esta investigación para extraer patrones y clasificarlos, con el fin de controlar remotamente un sistema 
electrónico. Por otro lado, en este artículo se demostró que son más relevantes los rangos de amplitudes de las señales en un espacio original, comparadas con las componentes de frecuencia obtenidas usando TDC y $\mathrm{TF}$; estos resultados tienen sentido, ya que las señales registradas tienen un comportamiento muy variante en el tiempo, y, así mismo, las componentes de frecuencia no se mantienen en rangos específicos, aunque se realice el mismo movimiento por el mismo sujeto.

Usando la TW se obtuvieron resultados que permiten concluir que es la más indicada para realizar la extracción de patrones, comparada con el ACP, TF y TDC.

Nosotros recomendamos aumentar el número de canales de registro para tener un sistema de mapeo mucho más robusto y de esta manera lograr elevar la precisión en el control del brazo. En cuanto al número de movimientos, también se propone para futuras investigaciones que sean por lo menos 6 , porque en el movimiento de la extremidad superior actúan gran cantidad de músculos, y con los movimientos planteados en esta investigación no se logró cubrir esta totalidad.

Finalmente, el posicionamiento preciso del brazo electrónico está sujeto al entrenamiento del usuario y al procesamiento de las SEMG. Con este trabajo se puede plantear una investigación para controlar un brazo mecánico que mezcle sustancias tóxicas; realizar procesos de teleoperación, y parametrizar movimientos de las extremidades.

\section{REFERENCIAS}

[1] A.H. Al-Timemy, G. Bugmann, J. Escudero and N. Outram, N. "Classification of Finger Movements for the Dexterous Hand Prosthesis Control With Surface Electromyography". Biomedical and Health Informatics, IEEE Journal. Vol. 17, pp. 608-618. 2013.

[2] L. Jizhou, Z. Yongiin and Z. Yong-Ping. "An indirect method to estimate the force output of triceps surae muscle". Engineering in Medicine and Biology Society (EMBC), 36th Annual International Conference of the IEEE. pp. 58325835. 2014.
[3] Z. Xiaodong and L. Haojie. "Study on Measurement and Processing Technology of Electromyography". Electronic Measurement and Instruments. pp. 1033-1036. 2007.

[4] R. Liye, W. Xiaoli and W. Xiao. "Research of Feature Extraction Method for Stroke Patients' Surface Electromyography". Intelligent Networks and Intelligent Systems (ICINIS). pp. 322-324. 2012.

[5] L. Zhizeng, W. Fei and M. Wenjie. "Pattern Classification of Surface Electromyography Based on AR Model and High-order Neural Network". Mechatronic and Embedded Systems and Applications. pp 1-6. 2006.

[6] G. Yang and S. Yang. "Emotion Recognition of Electromyography Based on Support Vector Machine". Intelligent Information Technology and Security Informatics (IITSI). pp. 298-301. 2010.

[7] J. Taelman, S. Van Huffel and A. Spaepen, "Wavelet-Independent Component Analysis to Remove Electrocardiography Contamination in Surface Electromyography". Engineering in Medicine and Biology Society. pp 682-685. 2007.

[8] K. Kuribayashi, S. Shimizu, K. Okimura and T. Taniguchi, "A discrimination system using neural network for EMG-controlled prosthesesIntegral type of EMG signal processing". Intelligent Robots and Systems '93, IROS '93. Proceedings IEEE/RSJ International Conference, vol. 3 (26), pp.1750-1755. 1993.

[9] A. Salman, J. Iqbal, U. Izhar, U.S. Khan and N. Rashid, "Optimized circuit for EMG signal processing". Robotics and Artificial Intelligence (ICRAI), 2012 International Conference. pp. 208-213. 2012.

[10] L. Marie-Françoise, S. Adrien Gaufriau, D. F. ChristianDoncarli. "Multi-channel surface EMG classification using support vector machines and signal-based wavelet optimization", Biomedical Signal Processing and Control, Vol. 3. 2008.

[11] J.L.G. Nielsen, S. Holmgaard, J. Ning Jiang; K. Englehart, D. Farina, P. Parker, "Enhanced EMG signal processing for simultaneous and proportional myoelectric control". Engineering in Medicine and Biology Society, 2009. EMBC 2009. Annual International Conference of the IEEE, pp. 4335-4338, Sept. 2009. 
[12] K. HeeSu, R. Kiwon, Y. Kyoung-jin, S. Hyun-chool, "Intuitive robot navigation using wireless EMG and acceleration sensors on human arm". Intelligent Signal Processing and Communications Systems (ISPACS), 2011 International Symposium, vol. 7(9), pp. 1-4 Dec. 2011.

[13] W. Xinqing, L. Yiwei, Y. Dapeng, L. Nan, L. Jiang, L. Hong, "Progress in the biomechatronic design and control of a hand prosthesis". Intelligent Robots and Systems (IROS), 2010 IEEE/RSJ International Conference, pp. 58805885. 2010.

[14] J. Z. Wang, R.C. Wang, F. Li, M. W. Jiang, D. W. Jin, "EMG Signal Classification for Myoelectric Teleoperating a Dexterous Robot Hand". Engineering in Medicine and Biology Society, 2005. IEEE-EMBS 2005. 27th Annual International Conference, vol. 7(9), pp. 59315933, Jan. 2006.

[15] J.C. González-Ibarra, C. Soubervielle-Montalvo, O. Vital-Ochoa, H. G. Pérez-González, "EMG Pattern Recognition System Based on Neural Networks". Artificial Intelligence (MICAI), 11th Mexican International Conference. pp. 7174. 2012.

[16] Y. Zhi-guo, W. Zhi-zhong, R. Xiao-mei. "Joint application of feature extraction based on EMDAR strategy and multi-class classifier based on LS-SVM in EMG motion classification". Journal of Zhejiang University SCIENCE, vol. 8(8), pp. 1246-1255. 2007.

[17] M. Galli, V. Cimolin, V. Crugnola, L. Priano, F. Menegoni, C. Trotti, E. Milano, A. Mauro. "Gait pattern in myotonic dystrophy (Steinert disease): A kinematic, kinetic and EMG evaluation using 3D gait analysis". Journal of the Neurological Sciences. vol. 314(1-2), pp. 83-87. 2012.

[18] A. Subasi. "Classification of EMG signals using PSO optimized SVM for diagnosis of neuromuscular disorders". Computers in Biology and Medicine, vol. 43 (5), pp. 576-581. 2013.

[19] A. Alkan, M. Günay. "Expert Systems with Applications Identification of EMG signals using discriminant analysis and SVM classifier", vol. 39 (1), pp. 44-47. 2012. 